Research Article

\title{
Pulse Splitting for Harmonic Beamforming in Time-Modulated Linear Arrays
}

\author{
Lorenzo Poli, ${ }^{1}$ Toshifumi Moriyama, ${ }^{2}$ and Paolo Rocca ${ }^{1}$ \\ ${ }^{1}$ Department of Information Engineering and Computer Science, ELEDIA Research Center, University of Trento, \\ Via Sommarive 5, 38123 Trento, Italy \\ ${ }^{2}$ School of Engineering, Nagasaki University, 1-14 Bunkyomachi, Nagasaki-shi 852-8521, Japan
}

Correspondence should be addressed to Lorenzo Poli; lorenzo.poli@disi.unitn.it

Received 31 January 2014; Accepted 29 April 2014; Published 17 June 2014

Academic Editor: Shiwen Yang

Copyright (C) 2014 Lorenzo Poli et al. This is an open access article distributed under the Creative Commons Attribution License, which permits unrestricted use, distribution, and reproduction in any medium, provided the original work is properly cited.

\begin{abstract}
A novel strategy for harmonic beamforming in time-modulated linear arrays is proposed. The pulse splitting technique is exploited to simultaneously generate two harmonic patterns, one at the central frequency and another at a preselected harmonic of arbitrary order, while controlling the maximum level of the remaining sideband radiations. An optimization strategy based on the particle swarm optimizer is developed in order to determine the optimal parameters describing the pulse sequence used to modulate the excitation weights of the array elements. Representative numerical results are reported and discussed to point out potentialities and limitations of the proposed approach.
\end{abstract}

\section{Introduction}

The synthesis of time-modulated antenna arrays is a topic that gained much attention from the scientific community in the last years. According to the principles conceived in [1], several strategies have been proposed aimed at determining suitable modulating functions defining the timevarying excitations of the elements in order to obtain desired radiation features. In this framework, many efforts have been devoted to synthesize a desired average pattern at the antenna working frequency while minimizing the spurious harmonic radiations (i.e., the sideband radiations, SRs [2]) caused by the modulation of the excitation amplitudes in the time domain. Toward this aim, several algorithms have been employed to find the optimal parameters of the modulating waveforms by solving the synthesis problem recast to an optimization one, among which are the simulated annealing (SA) [3], the genetic algorithms (GA) [4], the particle swarm optimizer (PSO) [5-9], the differential evolution (DE) [10-13], and the multiobjective differential evolution (MO-DEA) [14]. Although it has been shown that the SRs can be significantly reduced through a suitable shaping of the modulating functions [15], simpler periodic rectangular pulsed waveforms have been considered in all other works. Dealing with this latter kind of waveforms, conventional approaches consider a common rising instant for the pulses modulating the excitation weights $[13,16]$. However, the shifting of the rectangular pulses has been proposed in [17] as an additional degree of freedom in the synthesis process to properly control the harmonic radiations. Further degrees of freedom can be also introduced by considering split rectangular waveforms $[4,18-20]$. The division of the pulses into multiple shifted subpulses has been preliminarily investigated in [4], where the modulation period has been quantized into uniform time steps independently handled by a GA-based optimization technique. A more general case has been proposed in [18], where durations and rise instants of the rectangular subpulses have been arbitrarily defined by means of a DE-based optimization procedure. Moreover, suitable split rectangular waveforms have been also studied in [19] with the aim of shifting the undesired power on the SRs to obtain a partial suppression of the unwanted radiation by exploiting the limited bandwidth of the real radiating elements.

A first insight into the possibility of exploiting the pulse splitting to selectively distribute the power among 
the sideband harmonic frequencies was given in [20], where the pulses have been partitioned in two shifted parts in order to maximize the level of the second harmonic pattern, minimizing the level of the remaining harmonics while keeping the pattern at the working/central frequency unaltered. As a matter of fact, the pulse splitting enables the possibility of effectively synthesizing a desired pattern at an arbitrary harmonic frequency in order to take advantage of these sideband radiations for enabling advanced applications. Based on this perspective, some works have been recently proposed: multiple patterns have been simultaneously synthesized at different frequencies in [21-27] for direction of arrival estimation [21], adaptive beamforming [23, 24], and direction finding purposes $[23,26,27]$.

This paper is aimed at taking advantage of the pulse splitting approach in order to realize an easily reconfigurable antenna system able to provide a selective beamforming on the sideband patterns for a multichannel communication. A synthesis strategy based on the PSO is proposed to generate multiple harmonic beams aimed at simultaneously receiving multiple signals from different directions. Indeed, the harmonic patterns generated due to the time-modulation technique may be used as independent "channels" in the receiving mode that may be effectively handled through a proper combination of local oscillators and low-pass filters [25]. Dealing with the transmitting mode, the pulse splitting technique may also be exploited to synthesize a beam pattern at the harmonic frequency properly selected on the basis of the requirements arising from a working environment shared with other systems in order to transmit a signal on a free "channel" avoiding interferences.

The paper is organized as follows. The mathematical theory of the problem is formulated in Section 2, whereas the optimization strategy is described in Section 3. Selected numerical results are presented and discussed in Section 4. Some conclusions are finally remarked in Section 5 .

\section{Basic Mathematical Formulation}

Let us consider a linear array of $N$ elements aligned along the $z$-axis whose uniform excitation amplitudes vary in time according to a periodic modulation function $C(t)$ with period $T_{p}$, composed of $Q$ rectangular pulses defining in which portions of the modulation period the elements are in active (on) or inactive (off) state, as shown in Figure 1. The timevarying array factor can be calculated as

$$
F(\theta, t)=e^{j \omega_{0} t} \sum_{n=1}^{N} C(t) e^{j \beta(n-1) d \cos (\theta)},
$$

where $\omega_{0}$ is the angular working frequency of the antenna, $\beta=2 \pi / \lambda$ is the wavenumber, $d$ is the interelement spacing, and $\theta \in\left[0^{\circ}: 180^{\circ}\right]$ degrees. By expanding $C(t)$ in a Fourier series, (1) becomes

$$
F(\theta, t)=e^{j \omega_{0} t} \sum_{n=1}^{N}\left\{\sum_{h=-\infty}^{+\infty} c_{h n} e^{j \omega_{p} t}\right\} e^{j k(n-1) d \cos (\theta)},
$$

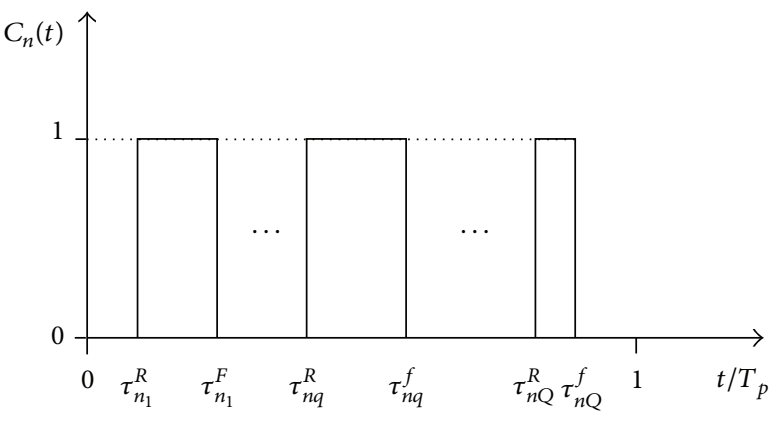

FIGURE 1: Example of split pulse controlling a RF switch.

where $\omega_{p}=2 \pi / T_{p}$ and the complex Fourier coefficients $c_{h n}$ $(h \in \mathbb{Z})$ can be computed as follows:

$$
c_{h n}=\frac{1}{T_{p}} \int_{0}^{T_{p}} C(t) e^{-j h \omega_{p} t} d t=\frac{j}{2 \pi h} \sum_{q=1}^{Q}\left[e^{j h \omega_{p} \tau_{n q}^{F}}-e^{j h \omega_{p} \tau_{n q}^{R}}\right],
$$

where $\tau_{n q}^{R}$ and $\tau_{n q}^{F}$ are the rise instant and the fall instant normalized with respect to the modulation period $T_{p}$ of the $q$ th subpulse related to $n$th waveform, respectively $(q=$ $1, \ldots, Q$ and $n=1, \ldots, N)$. The pattern generated in far-field at the angular frequency $\omega_{h}=\left(\omega_{0}+h \omega_{p}\right)$ is expressed as

$$
\left.F(\theta)\right|_{\omega_{h}}=\sum_{n=1}^{N} c_{h n} e^{j k(n-1) d \cos (\theta)} .
$$

Dealing with the central angular frequency $\omega_{0}$, it can be easily proved that the array factor $\left.F(\theta)\right|_{\omega_{0}}$ turns out to be

$$
\left.F(\theta)\right|_{\omega_{0}}=\sum_{n=1}^{N}\left[\sum_{q=1}^{Q}\left(\tau_{n q}^{F}-\tau_{n q}^{R}\right)\right] e^{j k(n-1) d \cos (\theta)} .
$$

\section{PSO-Based Synthesis Strategy}

The optimization strategy is aimed at simultaneously synthesizing a beam pattern at the working angular frequency $\omega_{0}$ oriented along the broadside direction $\theta^{\omega_{0}}=90^{\circ}$ and a beam pattern at a selected frequency $\omega_{\widehat{h}}=\left(\omega_{0}+\widehat{h} \omega_{p}\right)$, $\widehat{h} \in \mathbb{Z}_{0}$, steered toward an arbitrary direction $\theta^{\omega_{\hat{h}}} \in\left[0^{\circ}: 180^{\circ}\right]$. The PSO algorithm which has already provided effective results in the synthesis of time-modulated arrays [5-9, 23] has been applied to determine the optimized split pulse sequences exciting the uniform weights of the array elements. The parameters describing such pulse sequences, pictorially described in Figure 1, are the rise and the fall instants of the rectangular subpulses normalized to $T_{p}, \underline{\tau}^{R}=\left\{\tau_{n q}^{R} ; n=\right.$ $1, \ldots, N ; q=1, \ldots, Q\}$ and $\underline{\tau}^{F}=\left\{\tau_{n q}^{F} ; n=1, \ldots, N ; q=\right.$ $1, \ldots, Q\}$, respectively. However, since the optimization must provide results subject to physical rules about the sequence of the subpulses [i.e., $\left(\tau_{n q}^{F} \geq \tau_{n q}^{R}\right) ; q=1, \ldots, Q, n=$ $1, \ldots, N$ and $\left.\left(\tau_{n q}^{R} \geq \tau_{n(q-1)}^{F}\right) ; q=2, \ldots, Q, n=1, \ldots, N\right]$ in order to simplify the implementation of the constraints to 
be considered during the optimization process, the following variables will be optimized: $\underline{\tau}^{D}=\left\{\tau_{n q}^{D} ; n=1, \ldots, N ; q=\right.$ $1, \ldots, Q\}$ and $\underline{\tau}^{S}=\left\{\tau_{n q}^{S} ; n=1, \ldots, N ; q=1, \ldots, Q\right\}$, which represent the subpulse durations and the temporal shift among two adjacent subpulses [i.e., $\tau_{n q}^{S}$ represents the time delay between the subpulses $q$ and $(q-1)$ or the delay between the subpulse $q$ and the origin if $q=1$ ] normalized to $T_{p}$, respectively. Accordingly, the constraints can be simply recast to $\left[\left(\tau_{n q}^{D} \geq 0\right)\right.$ and $\left.\left(\tau_{n q}^{S} \geq 0\right) ; q=1, \ldots, Q, n=1, \ldots, N\right]$. The total number of variables to be optimized turns out to be $X=2 N Q$.

(i) Step 1 (parameters setup). Consider the following:

(a) definition of the desired pattern features described in terms of sidelobe level ( $\left.\mathrm{SLL}_{\mathrm{trg}}^{\omega_{h}}, h=0, \widehat{h}\right)$ first null beam width $\left(\Theta_{\text {trg }}^{\omega_{h}}, h=0, \widehat{h}\right)$, desired peak level evaluated along $\theta^{\omega_{h}}\left(\mathrm{Peak}_{\text {trg }}^{\omega_{h}}, h=0, \widehat{h}\right)$, and undesired sideband level $\left(\mathrm{SBL}_{\text {trg }}^{\omega_{\bar{h}}}, \bar{h}=1, \ldots, H, \bar{h} \neq \widehat{h}\right)$;

(b) setting of the PSO parameters: number of agents $(M)$, maximum number of iterations $(K)$, inertial weight $(I)$, cognitive and social acceleration coefficients $\left(C_{1}\right.$ and $C_{2}$, resp.), and convergence threshold $(\chi)$;

(c) definition of the research ranges for the unknown variables: $\tau_{n q}^{D} \in\left[\tau_{n q}^{D, \min }: \tau_{n q}^{D, \max } ; n=1, \ldots, N ; q=\right.$ $1, \ldots, Q]$ and $\tau_{n q}^{S} \in\left[\tau_{n q}^{S, \min }: \tau_{n q}^{S, \max } ; n=1, \ldots, N ; q=\right.$ $1, \ldots, Q]$.

(ii) Step 2 (swarm initialization). The particles of the swarm are randomly generated except for a single particle whose entries are analytically determined in order to speed up the convergence of the optimization process as follows.

(a) The parameters $\underline{\tau}^{D}$ and $\underline{\tau}^{S}$ have been initially set to $\tau_{n q}^{D}=\widehat{\tau}^{D}$ and $\bar{\tau}_{n q}^{S}=\widehat{\tau}^{S}(n=1, \ldots, N ; q=$ $1, \ldots, Q)$, where $\left(\widehat{\tau}^{D} ; \widehat{\tau}^{S}\right)$ represents the couple of parameters which allows obtaining the maximum value of the ratio $\Gamma$ (see (7)), evaluated for a limited set of combinations of the trial parameters $\tau^{D} \epsilon$ $\left\{\left(\tau^{D, \text { max }}-\tau^{D, \text { min }}\right) m / D_{\text {trial }} ; m=1, \ldots, D_{\text {trial }}\right\}$ and $\tau^{S} \in$ $\left\{\left(\tau^{S, \max }-\tau^{S, \min }\right) m / S_{\text {trial }} ; m=1, \ldots, S_{\text {trial }}\right\}:$

$$
\left(\widehat{\tau}^{D} ; \widehat{\tau}^{S}\right)=\left(\tau^{D}, \tau^{S}\right) \Longleftrightarrow \max _{\left(\tau^{D}, \tau^{S}\right)}\{\Gamma\}
$$

being

$$
\Gamma=\frac{\mathrm{SBL}^{\omega_{\hat{h}}}}{\sum_{h=1, h \neq \widehat{h}}^{H} \mathrm{SBL}^{\omega_{h}}},
$$

where $\mathrm{SBL}^{\omega_{h}}$ is the maximum level of the pattern associated with $\omega_{h}$. (b) The split pulse sequence is then shifted in order to steer the beam pattern generated at the angular frequency $\omega_{\hat{h}}$ along the desired direction $\theta^{\omega_{\hat{h}}}$ :

$$
\begin{gathered}
\tau_{n q}^{D}=\widehat{\tau}^{D} \quad n=1, \ldots, N ; q=1, \ldots, Q \\
\tau_{n q}^{S}=\widehat{\tau}^{S}+\frac{(n-1) d_{\lambda} \cos \left(\theta^{\omega_{h}}\right)}{\widehat{h}} \quad n=1, \ldots, N ; \\
q=1, \ldots, Q,
\end{gathered}
$$

where $d_{\lambda}$ is the interelement distance expressed in wavelength.

(iii) Step 3 (optimization process). The cost function defined in the following will be iteratively computed in order to evaluate if the shape of the multibeam pattern of the optimized configuration meets the requirements defined through the target pattern parameters initialized in Step 1(a). It can be expressed as the summation of three main terms:

$$
\Psi^{(k)}\left(\underline{\tau}^{D}, \underline{\tau}^{S}\right)=\Psi_{D}^{(k)}\left(\underline{\tau}^{D}, \underline{\tau}^{S}\right)+\Psi_{U}^{(k)}\left(\underline{\tau}^{D}, \underline{\tau}^{S}\right)+\Psi_{P}^{(k)},
$$

where $\Psi_{D}^{(k)}$ is the term aimed at shaping the multibeam pattern according to the parameters SLL $\mathrm{L}_{\mathrm{trg}}^{\omega_{h}}, \Theta_{\mathrm{trg}}^{\omega_{h}}$, and Peak $k_{\text {trg }}^{\omega_{h}}$ $(h=0, \widehat{h})$ that is defined as follows:

$$
\begin{aligned}
& \Psi_{D}^{(k)}\left(\underline{\tau}^{D}, \underline{\tau}^{S}\right) \\
& \quad=\sum_{\gamma \in \Upsilon} \alpha_{\gamma}\left\{\sum_{h=0, \widehat{h}}\left[\left(\frac{\left|\gamma^{\omega_{h}}-\gamma_{\operatorname{trg}}^{\omega_{h}}\right|^{2}}{\left|\gamma_{\operatorname{trg}}^{\omega_{h}}\right|^{2}}\right) \mathscr{H}\left(\gamma^{\omega_{h}}-\gamma_{\text {trg }}^{\omega_{h}}\right)\right]\right\},
\end{aligned}
$$

where $\Upsilon=\{$ SLL, $\Theta$, Peak $\}$ and $\mathscr{H}$ is the Heaviside function, whose argument has inverted sign when $\gamma \leftarrow$ Peak; the term $\Psi_{U}^{(k)}$ is devoted to handling the undesired sideband radiation limiting the maximum sideband level:

$$
\begin{array}{r}
\Psi_{U}^{(k)}\left(\underline{\tau}^{D}, \underline{\tau}^{S}\right)=\alpha_{U} \max _{\theta, \bar{h}}\left\{\frac{\left.|F(\theta)|_{\omega_{\bar{h}}}\right|^{2}}{\left.|F(\theta)|_{\omega_{0}}\right|^{2}}\right\}, \quad \theta \in\left[0^{\circ}: 180^{\circ}\right] ; \\
\bar{h}=1, \ldots, H, \bar{h} \neq \widehat{h} .
\end{array}
$$

Finally, the term $\Psi_{P}^{(k)}=\alpha_{P} \mathscr{H}\left(\left[\Sigma_{q=1}^{\mathrm{Q}}\left(\tau_{n q}^{S}+\tau_{n q}^{S}\right)\right]-1\right)$ is a penalty factor introduced to discard the solutions which provide meaningless configurations (i.e., when $\left[\Sigma_{q=1}^{\mathrm{Q}}\left(\tau_{n q}^{S}+\tau_{n q}^{S}\right)>\right.$ 1]). Moreover, $\alpha_{\gamma}(\gamma \in \Upsilon), \alpha_{U}$, and $\alpha_{P}$ are real weighting coefficients and $k$ is the PSO iteration index.

\section{Numerical Results}

Let us consider an arrangement of $N=16$ elements displaced along the $z$-axis and equally spaced of $d=\lambda / 2$. In a preliminary example, the synthesis of a sum beam 


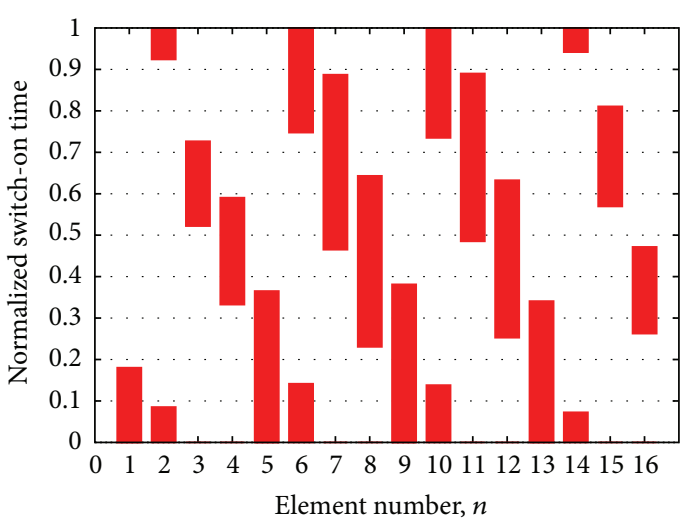

(a)

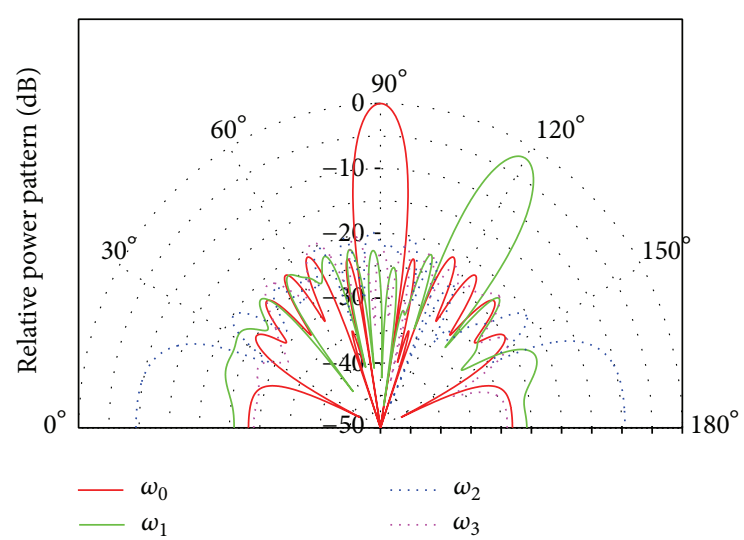

(b)

FIGURE 2: Single-pulse waveform, $Q=1$-multibeam sum pattern synthesis at $\omega_{0}$ and $\omega_{1}\left(N=16 ; d=\lambda / 2 ; \theta^{\omega_{0}}=90^{\circ} ; \theta^{\omega_{1}}=120^{\circ}\right)$ - plot of (a) the optimized pulse sequence and (b) corresponding power patterns generated at $\omega_{0}, \omega_{1}, \omega_{2}$, and $\omega_{3}$.

pattern at the central angular frequency $\omega_{0}$ in the broadside direction $\left(\theta^{\omega_{0}}=90^{\circ}\right)$ and a sum beam pattern at the fundamental frequency $\omega_{1}=\left(\omega_{0}+\omega_{p}\right)$ along $\theta^{\omega_{1}}=120^{\circ}$ is proposed. The specific multibeam configuration has been already investigated in [23], but in this case and unlike [23], in order to limit the loss of power, the reduction of the sideband level associated with the undesired harmonics $(|\bar{h}|=2, \ldots, H$, being in the specific case $H=10)$ has been also taken into account during the optimization process. The target pattern features have been selected as [23] $\mathrm{SLL}_{\mathrm{trg}}^{\omega_{0}}=$ $\mathrm{SLL}_{\text {trg }}^{\omega_{1}}=-20 \mathrm{~dB} ; \mathrm{SBL}_{\text {trg }}^{\omega_{1}}=-2 \mathrm{~dB} ;$ Peak $_{\operatorname{trg}}^{\omega_{1}}=-2.0 \mathrm{~dB}$; $\Theta_{\operatorname{trg}}^{\omega_{0}}=19.6^{\circ}, \Theta_{\operatorname{trg}}^{\omega_{1}}=20.6^{\circ}$ (these two latter have been chosen according to the optimized result achieved in [23], in order to take into account the unavoidable slight widening of the beam width due to the steering operation). Moreover, the undesired sideband level has been fixed to $\mathrm{SBL}_{\operatorname{trg}}^{\omega_{\bar{h}}}=-10 \mathrm{~dB}$. The parameters related to the optimization strategy have been selected according to [23], as well: $I=0.4, C_{1}=C_{2}=2.0$, $M=N, K=2000, \chi=10^{-5},\left[\tau_{n q}^{D, \min }: \tau_{n q}^{D, \text { max }}\right]=\left[\tau_{n q}^{S, \text { min }}:\right.$ $\left.\tau_{n q}^{S, \max }\right]=[0: 1]$; dealing with the cost function the weighting coefficients have been set to $\alpha_{\gamma}=\alpha_{U}=1(\gamma \in \Upsilon)$ and $\alpha_{P}=10^{3}$. Finally, in order to initialize the swarm for the PSO algorithm almost in real-time, the number of the trial solutions evaluated to analytically define one particle of the swarm has been fixed to $S_{\text {trial }} \times D_{\text {trial }}=20 \times 20=400$.

The power patterns generated at $\omega_{0}$ and $\omega_{1}$ afforded by the optimized pulse sequence of Figure 2(a) with $Q=1$ are shown in Figure 2(b). As expected, the optimization of durations and time-shifts of simple rectangular pulsed waveforms allows obtaining a desired multibeam radiation pattern that fulfills the given requirements, as can be seen from the data reported in Table 1 (i.e., $\mathrm{SLL}_{\mathrm{PSO}, \mathrm{Q}=1}^{\omega_{o}}=-21.06 \mathrm{~dB}$ versus $\mathrm{SLL}_{\text {trg }}^{\omega_{o}}=-20.00 \mathrm{~dB}$ and $\mathrm{SLL}_{\mathrm{PSO}, \mathrm{Q}=1}^{\omega_{1}}=-20.00 \mathrm{~dB}$ versus $\mathrm{SLL}_{\mathrm{trg}}^{\omega_{1}}=-20.00 \mathrm{~dB}$; Peak $\mathrm{PSO}, \mathrm{Q}=1^{\omega_{1}}=-2.0 \mathrm{~dB}$ versus Peak ${ }_{\text {trg }}^{\omega_{1}}=-2.0 \mathrm{~dB} ; \Theta_{\mathrm{PSO}, \mathrm{Q}=1}^{\omega_{0}}=18.8^{\circ}$ versus $\Theta_{\operatorname{trg}}^{\omega_{0}}=19.6^{\circ}$ and $\Theta_{\mathrm{PSO}, \mathrm{Q}=1}^{\omega_{1}}=20.6$ versus $\Theta_{\mathrm{trg}}^{\omega_{1}}=20.6^{\circ}$ ). Moreover,

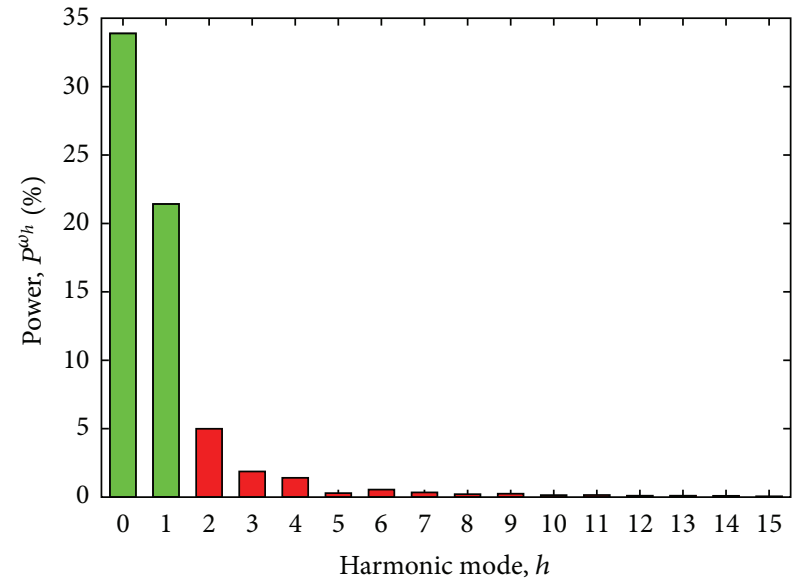

Figure 3: Single-pulse waveform, $Q=1-$ multibeam sum pattern synthesis at $\omega_{0}$ and $\omega_{1}\left(N=16 ; d=\lambda / 2 ; \theta^{\omega_{0}}=90^{\circ} ; \theta^{\omega_{1}}=\right.$ $120^{\circ}$ ) - plot of the power associated with the harmonic radiations in percentage on the total power.

the additional term in the cost function defined in (11) and aimed at controlling the undesired harmonic radiations allowed obtaining an improvement in terms of sideband level $\left(\mathrm{SBL}_{\mathrm{PSO}, \mathrm{Q}=1}^{\omega_{\bar{h}}}=-9.5 \mathrm{~dB}\right.$ versus $\mathrm{SBL} \frac{\omega_{\bar{h}}}{\mathrm{PSO}}=-6.44 \mathrm{~dB}$, where the subscript $\overline{\mathrm{PSO}}$ is related to the technique presented in [23]) and also in terms of useful power (expressed in percentage on the total radiated power) associated with the pattern synthesized at $\omega_{0}$ and $\omega_{1}$ as can be seen in Figure 3 (i.e., $P_{\mathrm{PSO}, \mathrm{Q}=1}^{\omega_{0}}=33.88 \%$ versus $P_{\mathrm{PSO}}^{\omega_{0}}=30.50 \%$ and $P_{\mathrm{PSO}, \mathrm{Q}=1}^{\omega_{1}}=$ $21.41 \%$ versus $\frac{P_{1}}{\omega_{1}}=20.70 \%$ ), in spite of a lower level of the pattern generated at the fundamental frequency $\omega_{1}$ $\left(\mathrm{SBL}_{\mathrm{PSO}, \mathrm{Q}=1}^{\omega_{1}}=-2 \mathrm{~dB}\right.$ versus $\left.\mathrm{SBL}_{\frac{\mathrm{PSO}}{\omega_{1}}}^{\omega_{1}}=-1.5 \mathrm{~dB}\right)$.

The synthesis problem has been addressed also considering split pulsed waveforms, setting $Q=2$ or $Q=3$, but in the specific case no significant improvements have been obtained with respect to the result reached with the 
TABLE 1: Multibeam sum pattern synthesis at $\omega_{0}$ and $\omega_{1}\left(N=16 ; d=\lambda / 2 ; \theta^{\omega_{0}}=90^{\circ} ; \theta^{\omega_{1}}=120^{\circ}\right)$ and pattern features for $Q=1, Q=2$, and $Q=3$.

\begin{tabular}{|c|c|c|c|c|c|c|c|c|}
\hline & $\mathrm{SLL}^{\omega_{0}}[\mathrm{~dB}]$ & $\mathrm{SLL}^{\omega_{1}}[\mathrm{~dB}]$ & $\Theta^{\omega_{0}}[\mathrm{deg}]$ & $\Theta^{\omega_{1}}[\mathrm{deg}]$ & Peak $^{\omega_{1}}[\mathrm{~dB}]$ & $\mathrm{SBL}^{\omega_{\bar{h}}}[\mathrm{~dB}]$ & $P^{\omega_{0}}[\%]$ & $P^{\omega_{1}}[\%]$ \\
\hline$Q=1 ; \omega_{0} \cap \omega_{1}$ & -21.06 & -20.00 & 18.8 & 20.6 & -2.0 & -9.50 & 33.88 & 21.41 \\
\hline$Q=2 ; \omega_{0} \cap \omega_{1}$ & -21.70 & -20.00 & 18.2 & 20.6 & -2.0 & -10.00 & 30.41 & 19.33 \\
\hline$Q=3 ; \omega_{0} \cap \omega_{1}$ & -21.21 & -20.00 & 18.2 & 20.6 & -2.0 & -9.80 & 31.48 & 19.90 \\
\hline
\end{tabular}

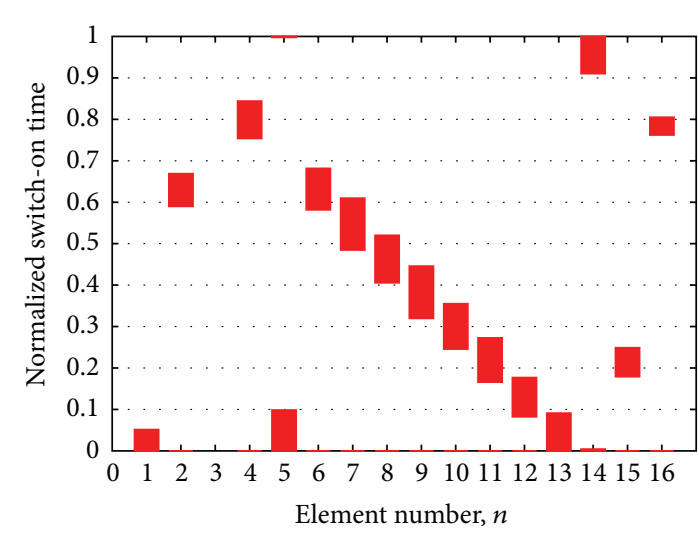

(a)

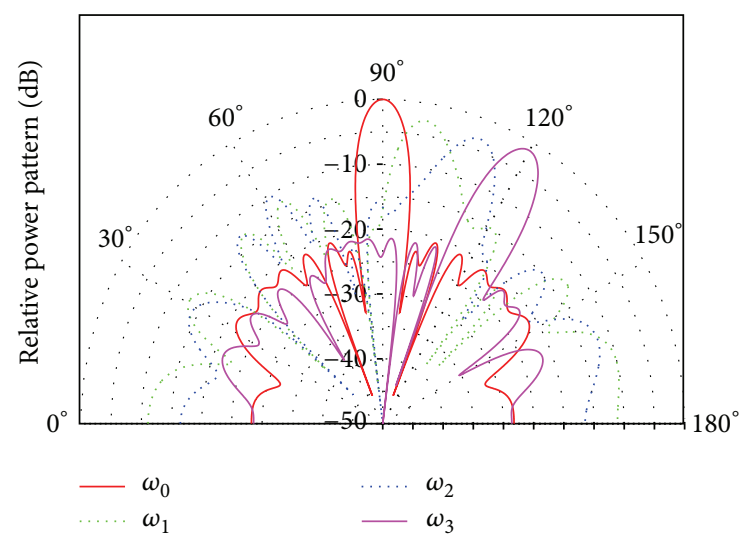

(b)

FIGURE 4: Single-pulse waveform, $Q=1$-multibeam sum pattern synthesis at $\omega_{0}$ and $\omega_{3}\left(N=16 ; d=\lambda / 2 ; \theta^{\omega_{0}}=90^{\circ} ; \theta^{\omega_{3}}=120^{\circ}\right)$ - plot of (a) the optimized pulse sequence and (b) corresponding power patterns generated at $\omega_{0}, \omega_{1}, \omega_{2}$, and $\omega_{3}$.

single-pulse rectangular waveforms. In fact, Table 1 shows that the configurations achieved for $Q=2$ and $Q=3$ provide a pattern at $\omega_{0}$ with lower sidelobe level and narrower beam width (i.e., $\mathrm{SLL}_{\mathrm{PSO}, \mathrm{Q}=2}^{\omega_{o}}=-21.70 \mathrm{~dB}$ and $\mathrm{SLL}_{\mathrm{PSO}, \mathrm{Q}=3}^{\omega_{0}}=$ $-21.21 \mathrm{~dB}$ versus $\mathrm{SLL}_{\mathrm{PSO}, \mathrm{Q}=1}^{\omega_{0}}=-21.06 \mathrm{~dB} ; \Theta_{\mathrm{PSO}, \mathrm{Q}=2}^{\omega_{0}}=$ $\Theta_{\mathrm{PSO}, \mathrm{Q}=3}^{\omega_{0}}=18.2^{\circ}$ versus $\Theta_{\mathrm{PSO}, \mathrm{Q}=1}^{\omega_{0}}=18.8^{\circ}$ ), but such solutions bring a higher waste of power in the $\mathrm{SRs}$ (i.e., $\mathrm{SR}_{\mathrm{PSO}, \mathrm{Q}=2}=$ $50.3 \%$ and $\mathrm{SR}_{\mathrm{PSO}, \mathrm{Q}=3}=48.6 \%$ versus $\left.\mathrm{SR}_{\mathrm{PSO}, \mathrm{Q}=3}=44.7 \%\right)$.

Unlike the previous case, the single-pulse rectangular waveforms do not provide satisfactory results when synthesizing the second beam pattern at a harmonic angular frequency of higher order $(|h|>1)$. Let us consider in the second example the synthesis of two sum beam patterns at the angular frequencies $\omega_{0}$ and $\omega_{3}=\left(\omega_{0}+3 \cdot \omega_{p}\right),(\widehat{h}=3)$. The target pattern features have been selected as in the previous case $\left(\mathrm{SLL}_{\operatorname{trg}}^{\omega_{0}}=\mathrm{SLL}_{\operatorname{trg}}^{\omega_{3}}=-20 \mathrm{~dB} ; \mathrm{SBL}_{\operatorname{trg}}^{\omega_{3}}=-2 \mathrm{~dB} ; \Theta_{\operatorname{trg}}^{\omega_{0}}=19.6^{\circ}\right.$, $\Theta_{\operatorname{trg}}^{\omega_{3}}=20.6^{\circ}$, and $\mathrm{SBL}_{\operatorname{trg}}^{\omega_{\bar{h}}}=-10 \mathrm{~dB}$ ). The optimized solution and the associated radiation power patterns are reported in Figures 4(a) and 4(b), respectively. Although the beam patterns have been almost perfectly shaped according to the input requirements as shown in Figure 4(b), the undesired sideband level turns out to be very high, $\mathrm{SBL}_{\mathrm{PSO}, \mathrm{Q}=1}^{\omega_{\bar{h}}}=$ $-2.77 \mathrm{~dB}$. Indeed, as shown in Figure 5, most of the power is lost in the unusable radiation, and the useful power associated with the patterns synthesized at $\omega_{0}$ and $\omega_{3}$ is limited to a small percentage of the total amount $\left(P_{\mathrm{PSO}, \mathrm{Q}=1}^{\omega_{0}}+P_{\mathrm{PSO}, \mathrm{Q}=1}^{\omega_{3}}\right)=$ $9.9 \%+7.1 \%=17 \%$.

Dealing with the same synthesis problem, more effective results can be obtained by setting the number of subpulses to $Q=3$, considering split rectangular waveforms.

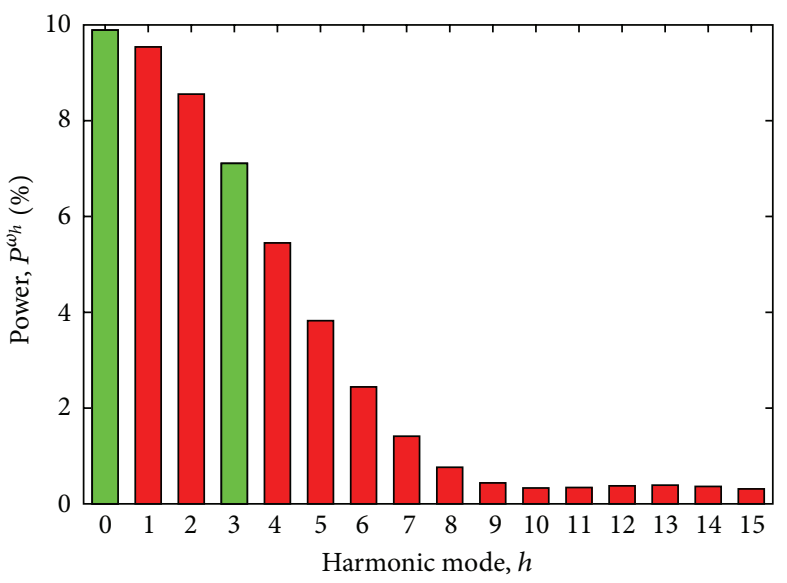

FIGURE 5: Single-pulse waveform, $Q=1-$ multibeam sum pattern synthesis at $\omega_{0}$ and $\omega_{3}\left(N=16 ; d=\lambda / 2 ; \theta^{\omega_{0}}=90^{\circ} ; \theta^{\omega_{3}}=\right.$ $120^{\circ}$ ) - plot of the power associated with the harmonic radiations in percentage on the total power.

The optimized pulse sequence turns out to be as in Figure 6(a) affording a multibeam radiation reported in Figure 6(b) that widely satisfies the initial requirements (Table 2). Figure 7(a) shows as the optimization of the split pulses allows selectively distributing the power among the harmonic frequencies, comparing the power associated with each harmonic when $Q=1$ (i.e., without splitting) and $Q=3$. Similar considerations arise from the observation of the comparison in terms of sideband level in Figure 7(b). As a matter of fact, by analyzing the undesired radiation at $\omega_{1}$ and $\omega_{2}$, 
TABLe 2: Multibeam sum pattern synthesis at $\omega_{0}$ and $\omega_{3}\left(N=16 ; d=\lambda / 2 ; \theta^{\omega_{0}}=90^{\circ} ; \theta^{\omega_{3}}=120^{\circ}\right)$ and pattern features for $Q=1, Q=2$, and $Q=3$.

\begin{tabular}{|c|c|c|c|c|c|c|c|c|}
\hline & $\mathrm{SLL}^{\omega_{0}}[\mathrm{~dB}]$ & $\mathrm{SLL}^{\omega_{3}}[\mathrm{~dB}]$ & $\Theta^{\omega_{0}}[\mathrm{deg}]$ & $\Theta^{\omega_{3}}[\mathrm{deg}]$ & Peak $^{\omega_{3}}[\mathrm{~dB}]$ & $\mathrm{SBL}^{\omega_{\bar{h}}}[\mathrm{~dB}]$ & $P^{\omega_{0}}[\%]$ & $P^{\omega_{3}}[\%]$ \\
\hline$Q=1 ; \omega_{0} \cap \omega_{3}$ & -20.81 & -20.00 & 18.6 & 20.6 & -1.4 & -2.77 & 9.89 & 7.11 \\
\hline$Q=2 ; \omega_{0} \cap \omega_{3}$ & -20.27 & -20.26 & 17.6 & 20.3 & -2.0 & -9.50 & 20.91 & 13.08 \\
\hline$Q=3 ; \omega_{0} \cap \omega_{3}$ & -20.95 & -20.37 & 17.4 & 20.1 & -2.0 & -10.00 & 31.28 & 19.60 \\
\hline
\end{tabular}

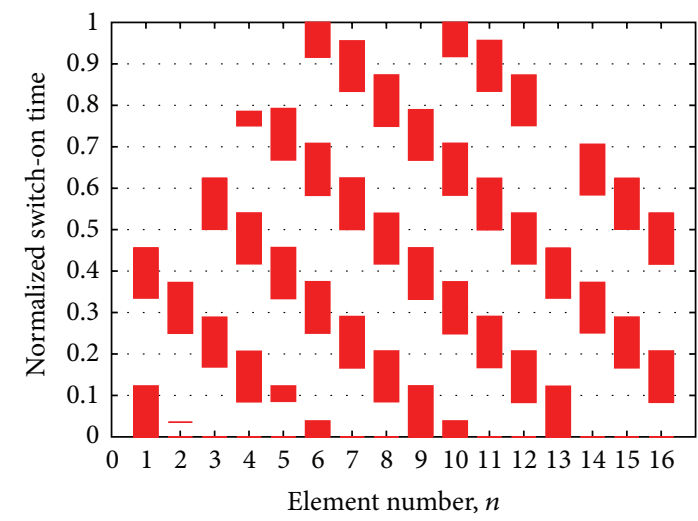

(a)

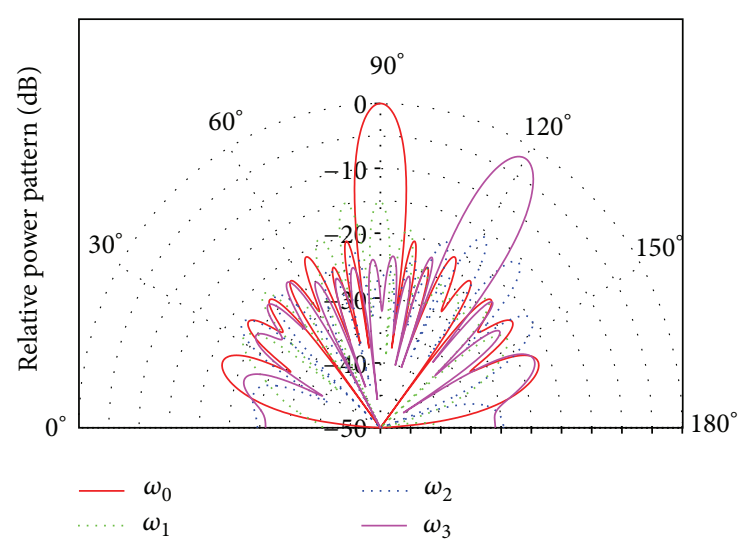

(b)

FIGURE 6: Multipulse waveform, $Q=3$-multibeam sum pattern synthesis at $\omega_{0}$ and $\omega_{3}\left(N=16 ; d=\lambda / 2 ; \theta^{\omega_{0}}=90^{\circ} ; \theta^{\omega_{3}}=120^{\circ}\right)-$ plot of (a) the optimized pulse sequence and (b) corresponding power patterns generated at $\omega_{0}, \omega_{1}, \omega_{2}$, and $\omega_{3}$.

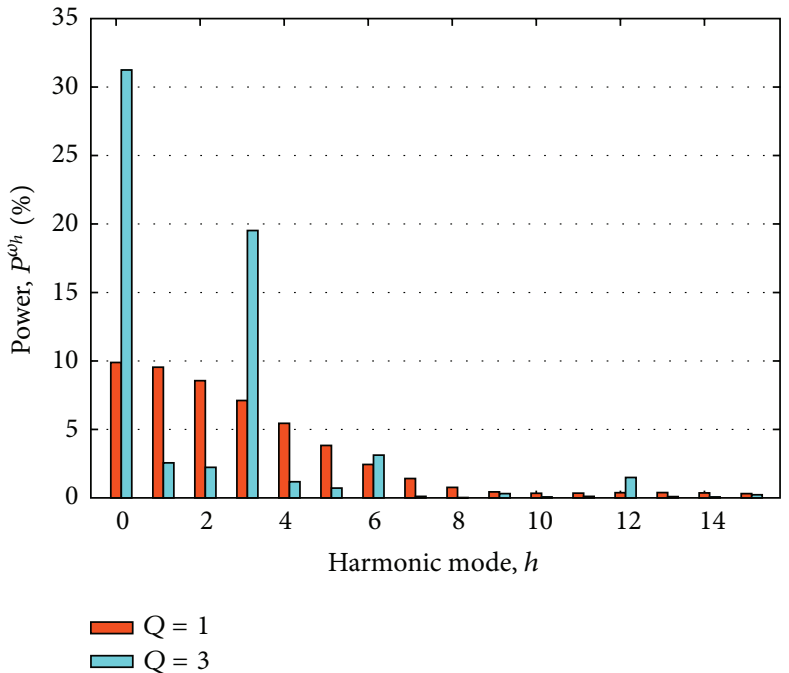

(a)

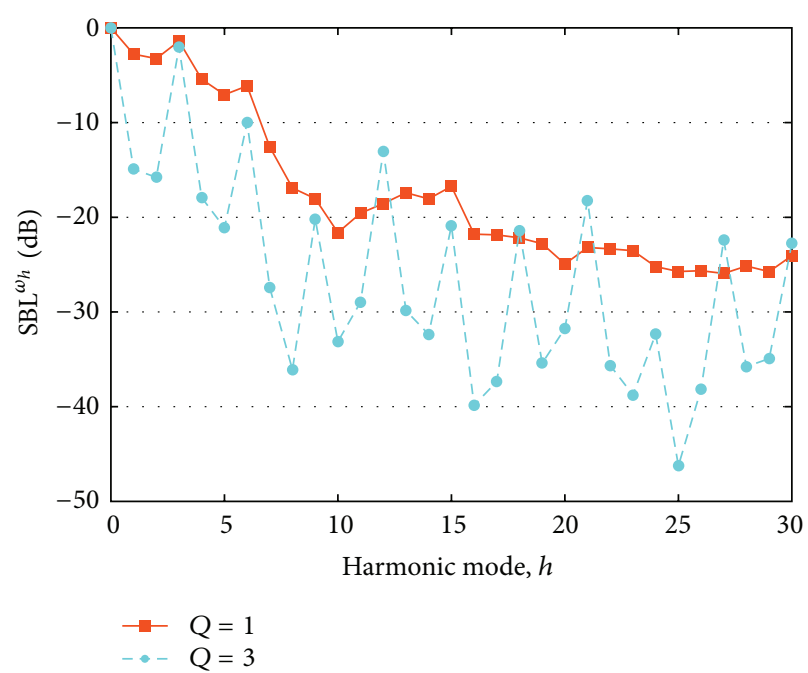

(b)

FIGURE 7: Comparative assessment-single-pulse waveform, $Q=1$ versus multipulse waveform, $Q=3\left(N=16 ; d=\lambda / 2 ; \theta^{\omega_{0}}=90^{\circ}\right.$; $\theta^{\omega_{3}}=120^{\circ}$ ) - plot of (a) the power associated with the harmonic radiations in percentage on the total power and (b) the sideband level associated with the harmonic patterns.

it is possible to observe that the sideband level decreases from $\mathrm{SBL}_{\mathrm{PSO}, \mathrm{Q}=1}^{\omega_{1}}=-2.77[\mathrm{~dB}]$ to $\mathrm{SBL}_{\mathrm{PSO}, \mathrm{Q}=3}^{\omega_{1}}=-14.92[\mathrm{~dB}]$ and from $\mathrm{SBL}_{\mathrm{PSO}, \mathrm{Q}=1}^{\omega_{2}}=-3.25[\mathrm{~dB}]$ to $\mathrm{SBL}_{\mathrm{PSO}, \mathrm{Q}=3}^{\omega_{2}}=-15.92[\mathrm{~dB}]$, respectively. Moreover, the maximum sideband level is also reduced of $\triangle S B L=\left|S L_{\mathrm{PSO}, \mathrm{Q}=1}-\mathrm{SBL}_{\mathrm{PSO}, \mathrm{Q}=3}\right|=\mid-10.00+$ $2.77 \mid=-7.23[\mathrm{~dB}]$.
However, it is worth noting that similar performance can be obtained avoiding the optimization of complex split rectangular waveforms. Indeed, starting from the optimized pulse sequence achieved in the preliminary example, described by the parameters $\widetilde{\tau}_{n q}^{R}$ and $\widetilde{\tau}_{n q}^{F}(n=1, \ldots, N ; q=1)$ which allows synthesizing the multibeam pattern at $\omega_{0}$ and 


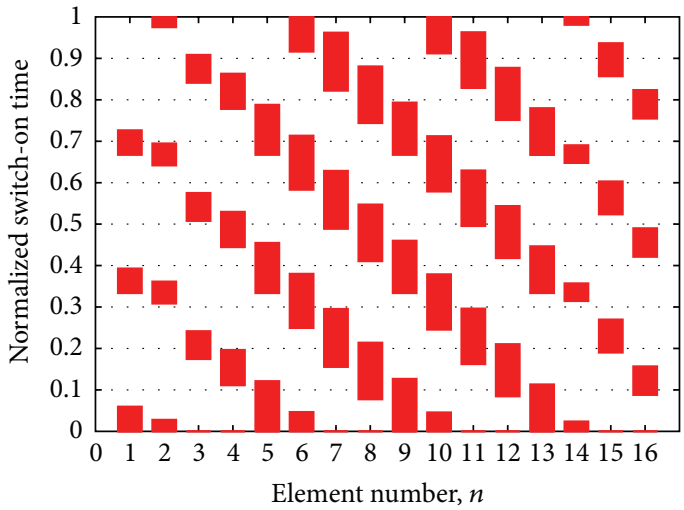

(a)

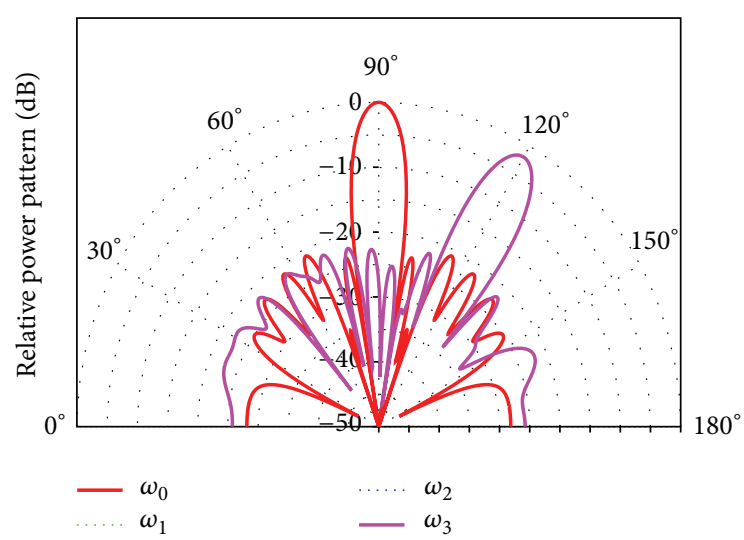

(b)

FIGURE 8: Multipulse waveform, $Q=3$-multibeam sum pattern synthesis at $\omega_{0}$ and $\omega_{3}\left(N=16 ; d=\lambda / 2 ; \theta^{\omega_{0}}=90^{\circ} ; \theta^{\omega_{3}}=120^{\circ}\right)$-plot of $(\mathrm{a})$ the analytically derived pulse sequence and (b) corresponding power patterns generated at $\omega_{0}, \omega_{1}, \omega_{2}$, and $\omega_{3}$.

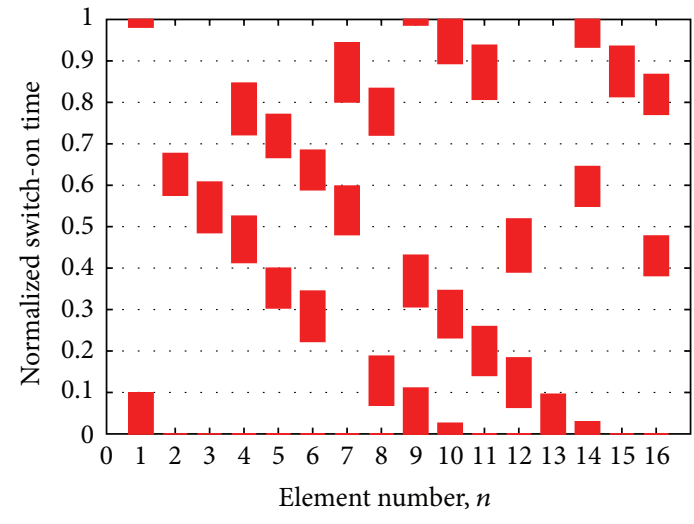

(a)

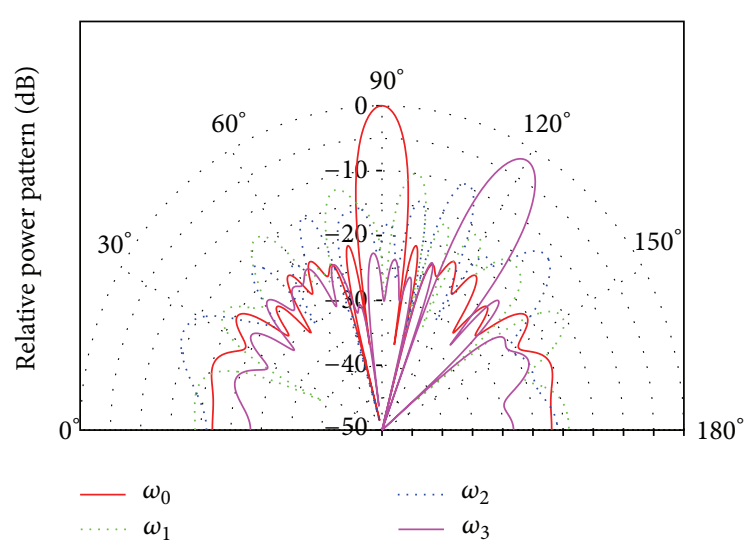

(b)

Figure 9: Multipulse waveform, $Q=2$-multibeam sum pattern synthesis at $\omega_{0}$ and $\omega_{3}\left(N=16 ; d=\lambda / 2 ; \theta^{\omega_{0}}=90^{\circ} ; \theta^{\omega_{3}}=120^{\circ}\right)$ - plot of (a) the optimized pulse sequence and (b) corresponding power patterns generated at $\omega_{0}, \omega_{1}, \omega_{2}$, and $\omega_{3}$.

$\omega_{1}=\left(\omega_{0}+\omega_{p}\right)$ of Figure 2(b), it is possible to easily derive the parameters of the split waveforms with $Q=\widehat{h}$ affording the generation of the second beam pattern at an arbitrary angular harmonic frequency $\omega_{\widehat{h}}=\left(\omega_{0}+\widehat{h} \cdot \omega_{p}\right)$, through

$$
\begin{array}{r}
\tau_{n q}^{R}=\frac{\tilde{\tau}_{n q}^{R}+(q-1)}{\widehat{h}}, \quad \tau_{n q}^{F}=\frac{\tilde{\tau}_{n q}^{F}+(q-1)}{\widehat{h}} \\
n=1, \ldots, N ; \quad q=1, \ldots, \widehat{h} .
\end{array}
$$

Therefore, the pattern previously generated at $\omega_{1}$ will be reproduced at $\omega_{\widehat{h}}$, nullifying the radiation at the frequencies $\omega_{h} \in\left\{\left(\omega_{0}+h \cdot \omega_{p}\right) ; h \neq q \cdot \widehat{h} ;|h|=1, \ldots, \infty ;|q|=0, \ldots, \infty\right\}$, but giving rise to a spreading of the harmonic spectrum (i.e., the pattern previously generated at $\omega_{h},|h|=1, \ldots, \infty$, will be shifted to $\left.\omega_{h \cdot \widehat{h}}\right)$. Dealing with the case for $\widehat{h}=3$, the analytically calculated parameters of the adapted split waveforms are reported in Figure 8(a), whereas the radiation patterns are shown in Figure 8(b).
However, (12) shows that the durations of the subpulses of the split waveforms depend on $\widehat{h}$, and more specifically the higher the harmonic index $\widehat{h}$, the shorter the durations of the subpulses. Accordingly, pulses with very short durations (i.e., very small fractions of the modulation period $T_{p}$ ) could arise from the necessity to shift the pattern to a harmonic frequency of high order (e.g., $\widehat{h}>3$ ) and could be hard to realize in practice. However, since the proposed optimization strategy does not require fixing the number of subpulses according to the harmonic index $\widehat{h}$ of the selected frequency, it can be profitably employed to synthesize the multibeam radiation previously investigated through simpler waveforms (i.e., with $Q=2<\widehat{h}$ ). In fact, the beam patterns shown in Figure 9(b) are generated by the split pulse sequence having a number of subpulses limited to $Q=2$ graphically described in Figure 9(a). At the expense of a higher amount of power lost in the SRs $\left(\mathrm{SR}_{\mathrm{PSO}, \mathrm{Q}=2}=66.0 \%\right.$ versus $\mathrm{SR}_{\overparen{\mathrm{PSO}}, \mathrm{Q}=3}=44.7 \%$, being the subscript $\widetilde{\mathrm{PSO}}$ related to 
the analytically derived solution of Figures $8(a)$ and $8(b))$, the optimization approach provides a solution characterized by a pulse sequence less complicated to implement, concerning the number of subpulses $\left(Q_{\mathrm{PSO}}=2\right.$ versus $\left.Q_{\overparen{\mathrm{PSO}}}=3\right)$ and the minimum duration of the pulses of the waveforms exciting the elements of the array (directly related to the maximum speed of the RF switches used to implement the modulating function $C(t))$, as well $\left(\min _{n, q}\left\{\tau_{n q}^{\mathrm{PSO}, \mathrm{Q}=2}\right\}=9.5 \times 10^{-2}\right.$ versus $\left.\min _{n, q}\left\{\tau_{n q}^{\mathrm{PSO}, \mathrm{Q}=3}\right\}=4.3 \times 10^{-2}\right)$.

\section{Conclusions}

A multibeam pattern synthesis technique has been proposed in this paper. A PSO-based strategy has been effectively employed to determine the optimal split pulse sequence aimed at synthesizing multiple patterns at different frequencies. The following considerations can be remarked from the analysis of the numerical results presented in the previous section.

(i) Multiple patterns can be simultaneously synthesized at the central frequency and at an arbitrarily selected harmonic frequency through the PSO-based strategy by applying the pulse splitting technique.

(ii) A higher index $\widehat{h}$ related to the synthesis of the second harmonic beam pattern requires more complicated waveforms to be implemented. The complexity of the split waveforms can be relaxed at expense of a higher amount of losses in the unused radiation.

Future efforts will be devoted to the extension of the proposed approach to the planar case and to study the possibility of defining alternative waveforms more suitable for the specific purpose.

\section{Conflict of Interests}

The authors declare that there is no conflict of interests regarding the publication of this paper.

\section{References}

[1] H. E. Shanks and R. W. Bickmore, "Four-dimensional electromagnetic radiators," Canadian Journal of Physics, vol. 37, pp. 263-275, 1959.

[2] J. C. Brégains, J. Fondevila-Gómez, G. Franceschetti, and F. Ares, "Signal radiation and power losses of time-modulated arrays," IEEE Transactions on Antennas and Propagation, vol. 56, no. 6, pp. 1799-1804, 2008.

[3] J. Fondevila, J. C. Brégains, F. Ares, and E. Moreno, "Optimizing uniformly excited linear arrays through time modulation," IEEE Antennas and Wireless Propagation Letters, vol. 3, no. 1, pp. 298301, 2004.

[4] S. Yang, Y. B. Gan, A. Qing, and P. K. Tan, "Design of a uniform amplitude time modulated linear array with optimized time sequences," IEEE Transactions on Antennas and Propagation, vol. 53, no. 7, pp. 2337-2339, 2005.

[5] L. Poli, P. Rocca, L. Manica, and A. Massa, "Handling sideband radiations in time-modulated arrays through particle swarm optimization," IEEE Transactions on Antennas and Propagation, vol. 58, no. 4, pp. 1408-1411, 2010.

[6] L. Poli, P. Rocca, L. Manica, and A. Massa, “Time modulated planar arrays - analysis and optimisation of the sideband radiations," IET Microwaves, Antennas and Propagation, vol. 4, no. 9, pp. 1165-1171, 2010.

[7] P. Rocca, L. Poli, G. Oliveri, and A. Massa, "Synthesis of timemodulated planar arrays with controlled harmonic radiations," Journal of Electromagnetic Waves and Applications, vol. 24, no. 5-6, pp. 827-838, 2010.

[8] P. Rocca, L. Poli, G. Oliveri, and A. Massa, "Synthesis of subarrayed time modulated linear arrays through a multi-stage approach," IEEE Transactions on Antennas and Propagation, vol. 59, no. 9, pp. 3246-3254, 2011.

[9] L. Poli, P. Rocca, and A. Massa, "Sideband radiation reduction exploiting pattern multiplication in directive time-modulated linear arrays," IET Microwaves, Antennas and Propagation, vol. 6, no. 2, pp. 214-222, 2012.

[10] S. Yang, Y. B. Gan, and A. Qing, "Sideband suppression in timemodulated linear arrays by the differential evolution algorithm," IEEE Antennas and Wireless Propagation Letters, vol. 1, pp. 173$175,2002$.

[11] E. Aksoy and E. Afacan, "Thinned nonuniform amplitude time-modulated linear arrays," IEEE Antennas and Wireless Propagation Letters, vol. 9, pp. 514-517, 2010.

[12] S. K. Mandal, G. K. Mahanti, and R. Ghatak, "Differential evolution algorithm for optimizing the conflicting parameters in time- modulated linear array antennas," Progress In Electromagnetics Research B, no. 51, pp. 101-118, 2013.

[13] Q. Zhu, S. Yang, R. Yao, and Z. Nie, "Design of a low sidelobe 4D planar array including mutual coupling," Progress In Electromagnetics Research M, vol. 31, pp. 103-116, 2013.

[14] Y. Chen, S. Yang, and Z. Nie, "Improving conflicting specifications of time-modulated antenna arrays by using a multiobjective evolutionary algorithm," International Journal of Numerical Modelling, vol. 25, no. 3, pp. 205-215, 2012.

[15] E. T. Bekele, L. Poli, P. Rocca, M. D'Urso, and A. Massa, "Pulse-shaping strategy for time modulated arrays-analysis and design," IEEE Transactions on Antennas and Propagation, vol. 61, no. 7, pp. 3525-3537, 2013.

[16] W. H. Kummer, A. T. Villeneuve, T. S. Fong, and F. G. Terrio, "Ultra-low sidelobes from time-modulated arrays," IEEE Transactions on Antennas and Propagation, vol. 11, no. 6, pp. 633-639, 1963.

[17] L. Poli, P. Rocca, L. Manica, and A. Massa, "Pattern synthesis in time-modulated linear arrays through pulse shifting," IET Microwaves, Antennas and Propagation, vol. 4, no. 9, pp. 1157$1164,2010$.

[18] Q. Zhu, S. Yang, L. Zheng, and Z. Nie, "Design of a low sidelobe time modulated linear array with uniform amplitude and subsectional optimized time steps," IEEE Transactions on Antennas and Propagation, vol. 60, no. 9, pp. 4436-4439, 2012.

[19] Y. Tong and A. Tennant, "Sideband level suppression in timemodulated linear arrays using modified switching sequences and fixed bandwidth elements," Electronics Letters, vol. 48, no. 1, pp. 10-11, 2012.

[20] E. Aksoy and E. Afacan, "Sideband level suppression improvement via splitting pulses in time modulated arrays under static fundamental radiation," in Proceedings of the Progress in Electromagnetics Research Symposium (PIERS '11), pp. 364-367, Suzhou, China, September 2011. 
[21] G. Li, S. Yang, and Z. Nie, "Direction of arrival estimation in time modulated linear arrays with unidirectional phase center motion," IEEE Transactions on Antennas and Propagation, vol. 58, no. 4, pp. 1105-1111, 2010.

[22] G. Li, S. Yang, M. Huang, and Z. Nie, "Shaped patterns synthesis in time-modulated antenna arrays with static uniform amplitude and phase excitations," Frontiers of Electrical and Electronic Engineering in China, vol. 5, no. 2, pp. 179-184, 2010.

[23] L. Poli, P. Rocca, G. Oliveri, and A. Massa, "Harmonic beamforming in time-modulated linear arrays," IEEE Transactions on Antennas and Propagation, vol. 59, no. 7, pp. 2538-2545, 2011.

[24] Y. Tong and A. Tennant, "A two-channel time modulated linear array with adaptive beamforming," IEEE Transactions on Antennas and Propagation, vol. 60, no. 1, pp. 141-147, 2012.

[25] G. Li, S. Yang, Y. Chen, and Z. Nie, "A novel electronic beam steering technique in time modulated antenna arrays," Progress in Electromagnetics Research, vol. 97, pp. 391-405, 2009.

[26] A. Tennant and B. Chambers, "A two-element time-modulated array with direction-finding properties," IEEE Antennas and Wireless Propagation Letters, vol. 6, pp. 64-65, 2007.

[27] Y. Chen, S. Yang, and Z. Nie, "Design of a novel monopulse antenna system using the time-modulated antenna arrays," International Journal of $R F$ and Microwave Computer-Aided Engineering, vol. 20, no. 2, pp. 163-169, 2010. 

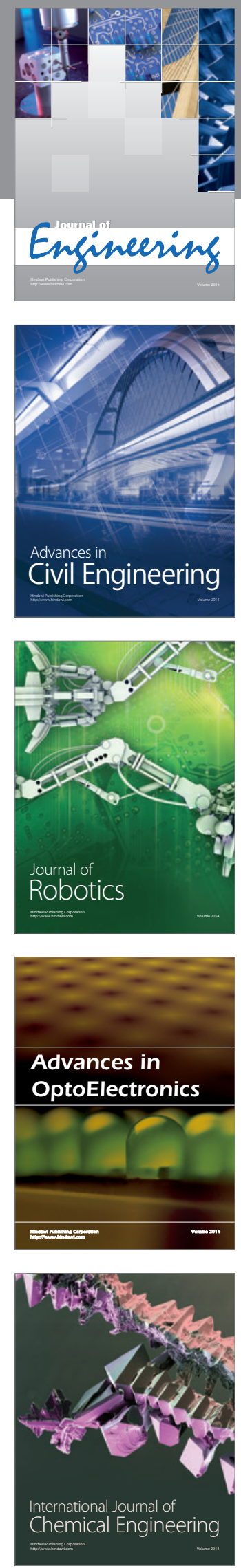

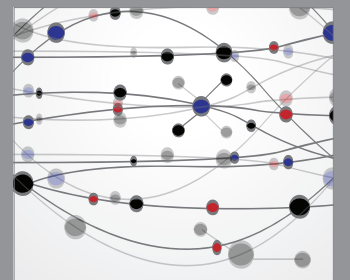

The Scientific World Journal
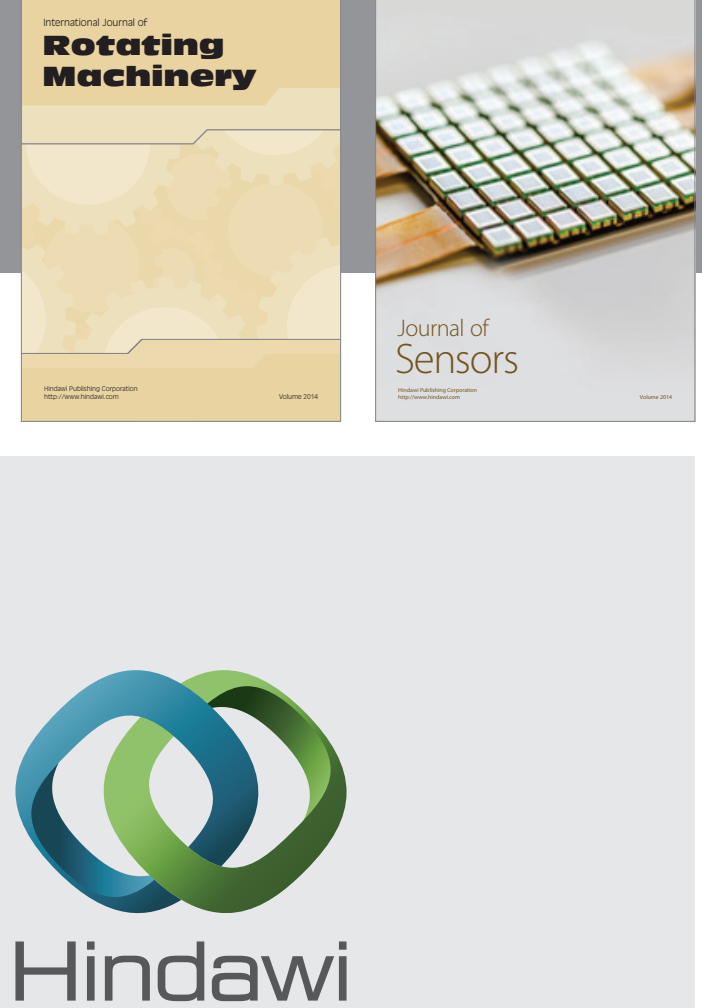

Submit your manuscripts at http://www.hindawi.com
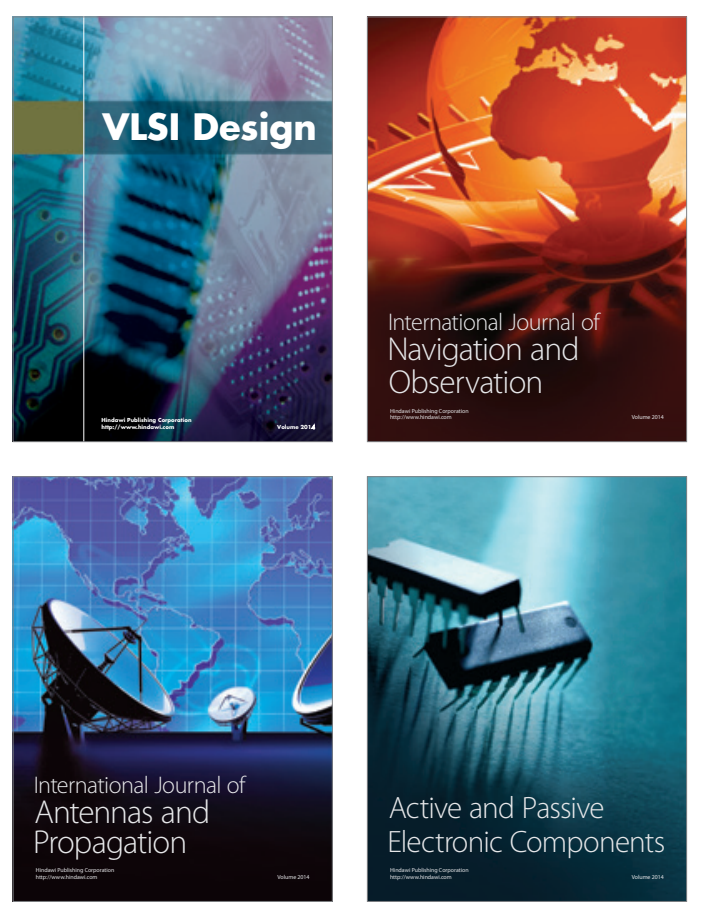
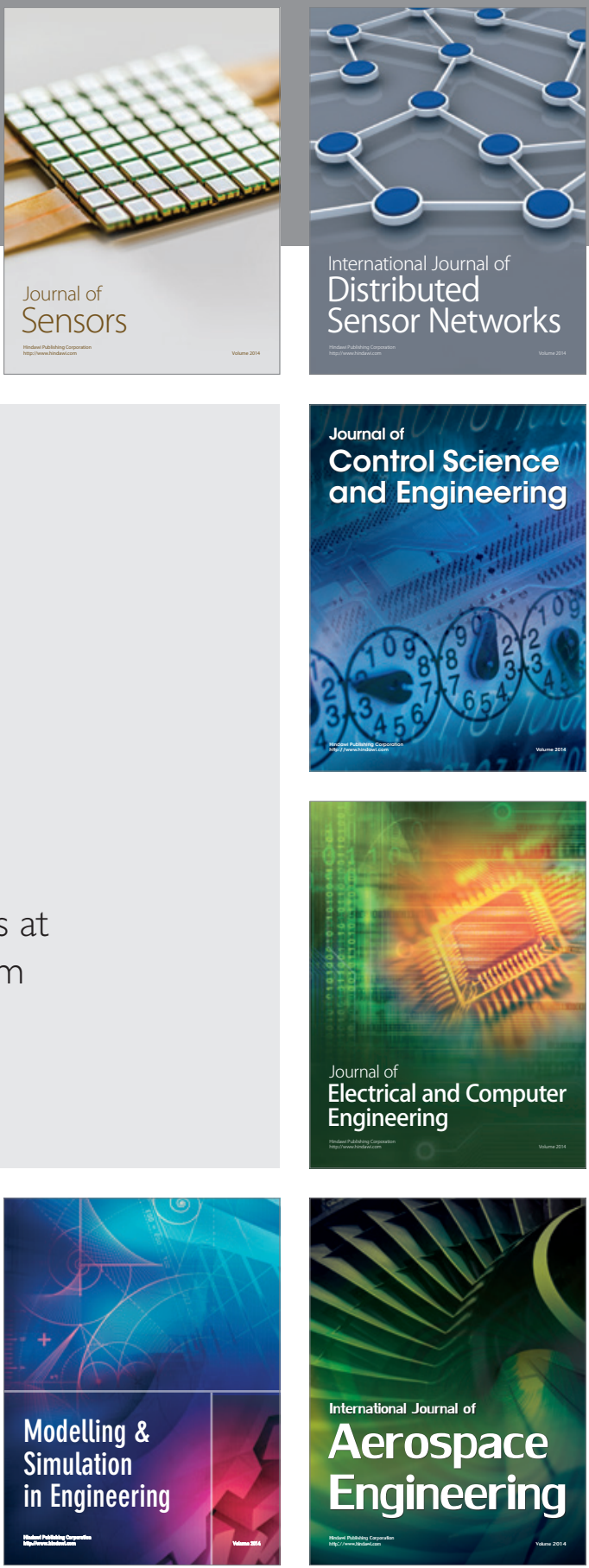

Journal of

Control Science

and Engineering
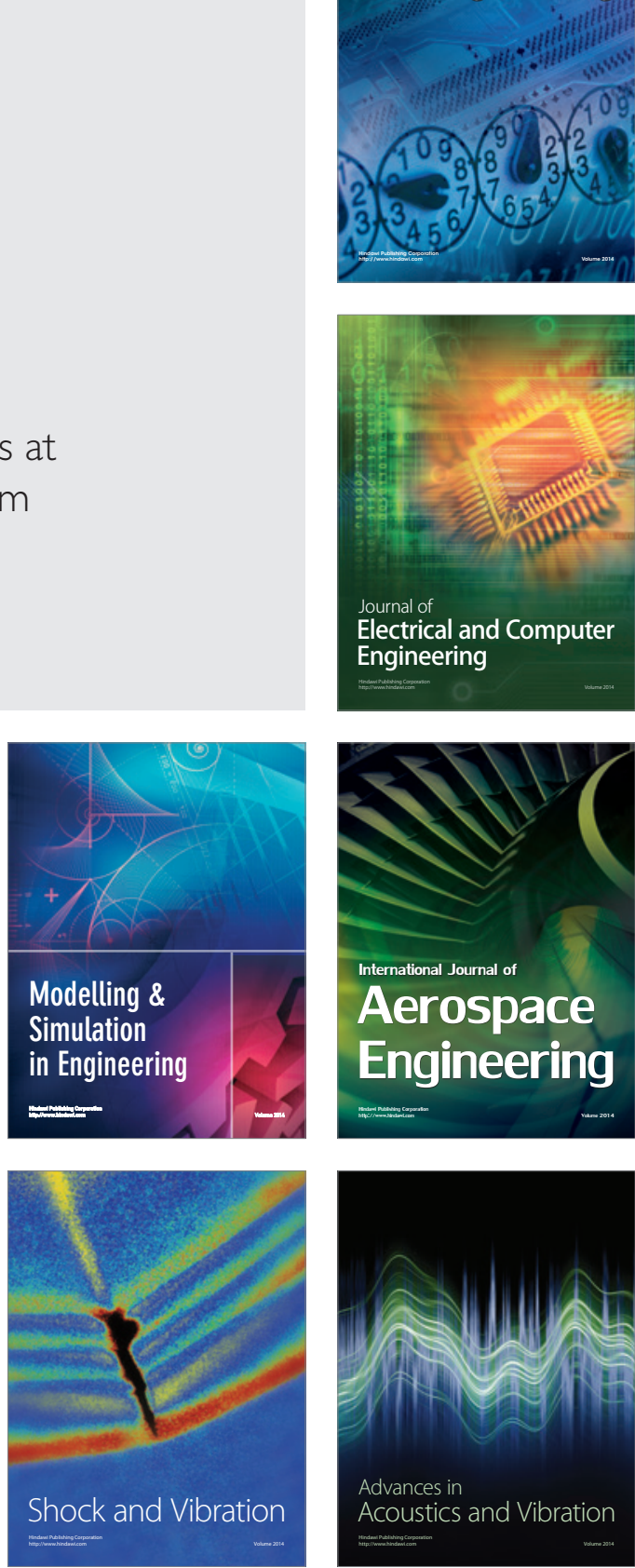